University of Wollongong

Research Online

Faculty of Engineering and Information

Faculty of Engineering and Information

Sciences - Papers: Part A

Sciences

$1-1-2015$

Stability enhancement of magnetic levitation ball system with two controlled electomagnets

Hai Huang

University of Wollongong, hh035@uowmail.edu.au

Haiping Du

University of Wollongong, hdu@uow.edu.au

Weihua Li

University of Wollongong, weihuali@uow.edu.au

Follow this and additional works at: https://ro.uow.edu.au/eispapers

Part of the Engineering Commons, and the Science and Technology Studies Commons

Research Online is the open access institutional repository for the University of Wollongong. For further information contact the UOW Library: research-pubs@uow.edu.au 


\title{
Stability enhancement of magnetic levitation ball system with two controlled electomagnets
}

\begin{abstract}
Magnetic levitation ball system is a highly nonlinear and unstable system. If the system is linearised at the equilibrium point, the achievable balance range of the magnetically levitated ball is very short with one electromagnet. This paper presents an improved magnetic levitation ball system, that contains two electromagnets, where one electromagnet is set above the magnetic ball for pulling, and another one is set under the magnetic ball for pushing. In addition, this paper applies a 2-DOF (degree-of-freedom) PID (proportional-integral-derivative) controller instead of 1-DOF PID for the control of the two electromagnets so that they can work corporately to make the magnetic ball levitate in the air. These improvements not only increase the balance range of the ball, but also make the magnetic levitation ball system more stable. The effectiveness of the proposed system is further validated by both numerical simulations and real experiments.
\end{abstract}

\section{Keywords}

two, electomagnets, levitation, magnetic, enhancement, system, stability, controlled, ball

\section{Disciplines}

Engineering | Science and Technology Studies

\section{Publication Details}

H. Huang, H. Du \& W. Li, "Stability enhancement of magnetic levitation ball system with two controlled electomagnets," in Power Engineering Conference (AUPEC), 2015 Australasian Universities, 2015, pp. 1-6. 


\title{
Stability Enhancement of Magnetic Levitation Ball System with Two Controlled Electomagnets
}

\author{
Hai Huang*, Haiping Du and Weihua Li \\ School of Electrical, Computer \& Telecommunications Engineering \\ The University of Wollongong \\ Wollongong, Australia. \\ *hh035@uowmail.edu.au
}

\begin{abstract}
Magnetic levitation ball system is a highly nonlinear and unstable system. If the system is linearised at the equilibrium point, the achievable balance range of the magnetically levitated ball is very short with one electromagnet. This paper presents an improved magnetic levitation ball system, that contains two electromagnets, where one electromagnet is set above the magnetic ball for pulling, and another one is set under the magnetic ball for pushing. In addition, this paper applies a 2DOF (degree-of-freedom) PID (proportional-integral-derivative) controller instead of 1-DOF PID for the control of the two electromagnets so that they can work corporately to make the magnetic ball levitate in the air. These improvements not only increase the balance range of the ball, but also make the magnetic levitation ball system more stable. The effectiveness of the proposed system is further validated by both numerical simulations and real experiments.
\end{abstract}

Keywords-Magnetic levitation ball system; 2-DOF PID control; electromagnet control.

\section{INTRODUCTION}

Because of the particular non-contact feature, the magnetic levitation system has been widely implemented and applied in many areas in the world from magnetically levitated vehicles to artworks [6]. For example, in [7], the magnetic levitation technology was used for the railway transportation system. Moreover, because there is no any mechanical contact and less heat generation, magnetic bearings were applied for blood pumps [8]. Another good application of the magnetic levitation system is the magnetic actuator [10]. The magnetic actuator can generate non-contact force to reduce the vibration transmission no matter it is high frequency vibration or low frequency vibration, so it can be well used for vibration isolation [9][10]. However, the magnetic levitation systems are quite unstable and nonlinear in the open-loop [2]. So, how to design a good structure and an effective controller is critical for the real applications of magnetic levitation systems.

The magnetic levitation ball system is a benchmark magnetic levitation system that can generate an appropriate magnetic force to make an iron ball suspend in the air by appropriately regulating the coil current [1]. This system is often used to study the system modelling and control issues so that any newly proposed schemes can be further applied to the real applications. Magnetic levitation ball systems mainly have two kinds of structures [1]. In the first structure, an electromagnet is put over the magnetic ball to provide an attractive force to counteract the gravity of the ball [3] [4]. So this structure is simple and efficient in energy consumption [4]. However, because of the nonlinear characteristics of the electromagnet, the controllable gap between the ball and the electromagnet is very small, and as the gap increases, the system becomes more and more unstable. Another structure is that an electromagnet is set under the ball to provide a repulsive force [5]. But in order to make ball suspend stably in this case, additional electromagnets need to provide forces on a plurality of directions to bind the ball. So, three or more electromagnets have to be used in this structure. However, these forces are not in one direction, the efficiency of the system is greatly decreased. Using a suitable structure in different applications is becoming a necessary condition.

Another necessary component for the successful application of the magnetic levitation system is the controller design. In recent years, a lot of scholars studied on how to better control the magnetic levitation systems based on the linearised model or the nonlinear model. In [12] a nonlinear feedback linearizing controller has been used for magnetic levitation systems [2]. And some researchers have used robust controller like Qparameterization theory and $\mu$-analysis control or $\mathrm{H}_{\infty}$ disturbance attenuation controller [13] [14]. In order to increase the suspension distance and achieve good tracking, other researchers consider the nonlinear models. More complex controllers are used for the control of the magnetic levitation systems, for example fuzzy logic controller, neural controllers, and fuzzy compensation based adaptive PID controller were proposed in the literature [15] [16] [18]. However most of these controllers are complex, and therefore, need high computational effort.

In this paper, a new magnetic levitation ball structure that uses two electromagnets corporately to make a magnetic ball suspend is presented. Using this structure, the distance between the ball and the electromagnets increases greatly, and as the balance range of the ball increases, some new applications can be achieved. For example, levitating disco ball can levitate at the centre of a dancing floor [19]. Furthermore, the stability of the system is increased when two forces work corporately. In 
addition, a 2-DOF PID controller is applied in this paper. Compared with the 1-DOF PID controllers which have been often used before, the 2-DOF PID controller has simple structure and is more efficient [20] [21]. Simulation results show that the magnetic levitation ball system can work as expected with the proposed structure and the designed 2-DOF PID controller. Furthermore, the real experiments, where a Hilink real-time control board is used as an interface between the real magnetic levitation ball system and the Matlab/Simulink software for the implementation of the hardware-in-the-loop control, confirm the results.

This paper is organised as following. Section II contains the mathematical model of the magnetic levitation ball system. Section III is devoted to the design of the controller for the system. Section IV presents the simulation and real experiment results of the magnetic levitation ball system. Finally, the conclusion is given in Section V.

\section{DYNAMIC MODELLING OF MAGNETIC LEVITATION BALL SYSTEM}

The proposed magnetic levitation ball system consists of two electromagnets that can work together to make a magnetic ball levitate. A hall-effect sensor is used for measuring the position of the magnet ball [15]. The two electromagnets, one is over the ball and another one is under the ball. The advantage of this structure is that both forces are in the same direction and the forces which are on other directions to be used for binding the ball are not needed any more.

Fig. 1 shows that the ball is subjected to three forces, pulling force $F_{1}$ generated by the coil $L_{1}$, repulsive force $F_{2}$ generated by the coil $\mathrm{L}_{2}$, and the gravity of the ball. Because of using two same electromagnets, the resistance $R_{1}=R_{2}$, the induction $\mathrm{L}_{1}=\mathrm{L}_{2}$. The displacement is $\mathrm{x}_{2}=\mathrm{X}-\mathrm{x}_{1}$, where $\mathrm{X}$ is the distance between $\mathrm{x}_{1}$ and $\mathrm{x}_{2}$, and the control voltage is $\mathrm{u}_{1}=\mathrm{k}_{1} \mathrm{u}_{2}$, where $\mathrm{k}_{1}$ is a positive constant. The hall-effect sensor equation, which is used to measure the position of the ball, is $\mathrm{x}_{1}=\mathrm{ce}$, where $\mathrm{c}$ is the parameter of the sensor, and $\mathrm{e}$ is the output of the sensor.

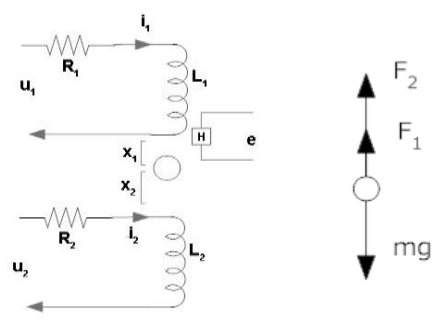

Fig. 1. Model of the proposed electromagnetic levitation ball system.

The summation of the two magnetic forces generated by the electromagnets can be described as:

$$
F(i, x)=F_{1}-F_{2}
$$

Then, the motion of the magnetic levitation ball is described by the following equation,

$$
m \ddot{x}=m g-F(i, x)
$$

Where $\mathrm{m}$ is the mass of the magnetic ball; $\mathrm{g}$ is gravity constant; $i$ is the current of the electromagnet coil current; $x$ is the displacement between the electromagnet and the ball; and

$$
F(i, x)=K \frac{i^{2}}{x^{2}}
$$

where $\mathrm{K}$ is a constant. When the ball is at the balance point, then $m \ddot{x}=0$, and,

$$
m g=K \frac{i_{0}^{2}}{x_{0}^{2}}
$$

For linearisation purpose, Taylor formula can be used:

$$
\begin{aligned}
& F_{i}\left(i_{0}, x_{0}\right)=\left.\frac{\delta F(i, x)}{\delta i}\right|_{i=i_{0} x=x_{0}} \\
& F_{x}\left(i_{0}, x_{0}\right)=\left.\frac{\delta F(i, x)}{\delta x}\right|_{i=i_{0} x=x_{0}}
\end{aligned}
$$

Then let $K_{i}$ as the current stiffness parameter and $K_{x}$ as the displacement stiffness parameter:

$$
\begin{gathered}
K_{i}=F_{i}\left(i_{0}, x_{0}\right)=\frac{2 K i_{0}}{x_{0}{ }^{2}} \\
K_{x}=F_{x}\left(i_{0}, x_{0}\right)=-\frac{2 K i_{0}}{x_{0}{ }^{3}}
\end{gathered}
$$

The system (2) about the equilibrium position becomes:

$$
m \frac{d^{2} x}{d t^{2}}=K_{i}\left(i-i_{0}\right)-K_{x}\left(x-x_{0}\right)
$$

The voltage-current relationships for the coils are given by:

$$
\begin{aligned}
& u_{1}(t)=R_{1} i_{1}(t)+L_{1} \frac{d i_{1}(t)}{d t} \\
& u_{2}(t)=R_{2} i_{2}(t)+L_{2} \frac{d i_{2}(t)}{d t}
\end{aligned}
$$

Because at the balance point, the value of the di(t)/dt is very small, so $i_{1}$ is approximately equal to $\mathrm{k}_{1} \mathrm{i}_{2}$.

Although with increasing the $\mathrm{x}$, the accuracy of the equation (9) reduces, the practice proves that the method of linearisation is appropriate in many applications on the controller design. After doing the Laplace transform, (9) becomes:

$$
\mathrm{x}(\mathrm{s}) s^{2}=\frac{2 K i_{0}}{x_{0}{ }^{2}} i(s)-\frac{2 K i_{0}}{x_{0}{ }^{3}} x(s)
$$

By using (4), the following system transfer function is obtained

$$
\frac{\mathrm{x}(\mathrm{s})}{\mathrm{i}(s)}=\frac{-1}{A s^{2}-B}
$$

where $\mathrm{A}=\mathrm{i}_{0} / 2 \mathrm{~g}, \mathrm{~B}=\mathrm{i}_{0} / \mathrm{x}_{0}$. 
If defining the input of the control plant as voltage of the amplifier $U_{i n}$, the output of the system $x$ as voltage of the output $\mathrm{U}_{\text {out }}$ (the output voltage of the sensor), the system openloop transfer function becomes:

$$
\mathrm{G}(\mathrm{s})=\frac{U_{\text {out }}(s)}{U_{\text {in }}(s)}=\frac{K_{s} x(s)}{K_{a} i(s)}=-\frac{K_{S}}{K_{a}\left(A s^{2}-B\right)}
$$

where, $K_{s}$ is the parameter of the sensor, $K_{a}$ is D/A output amplifier parameter.

\section{CONTROLLER DESIGN}

A feed-forward type 2-DOF PID controller is used in this study. Although 1-DOF PID controller has simple structure and less parameters to be adjusted, it cannot well control the magnetic levitation ball system, and even may cause larger overshoots and reduce the stability of the system due to the system`s highly nonlinear and unstable characteristics. Unlike 1-DOF PID controller, a feed-forward gain presented in the 2DOF structure can be used to obtain superior plant output and control responses effectively. When the system`s order is more than two or system has time delay, 2-DOF PID controller can be more effective. It is noted that there exist other types of 2DOF PID structures, which are effective for some specific applications. But in the proposed magnetic levitation ball system, only the feed-forward type 2-DOF PID controller is discussed.

\section{A. Structure of the feed-forward 2-DOF PID controller}

The structure of the 2-DOF PID controller for the magnetic levitation ball system is shown in Fig. 2.

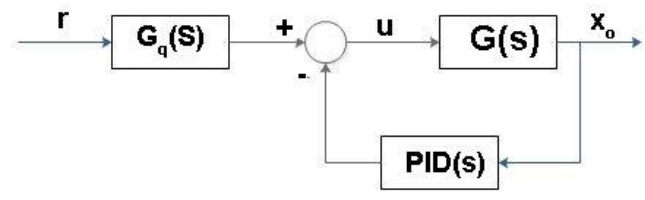

Fig. 2. The structure of the 2-DOF PID controller.

In the 2-DOF PID controller, the feed-forward part is defined as

$$
G_{q}(s)=\frac{q_{1}}{s}+q_{2}
$$

where the additional gain parameters $\mathrm{q}_{1}$ and $\mathrm{q}_{2}$ help to add a zero at desired location to achieve superior responses. The feedback part is defined as

$$
\operatorname{PID}(s)=k_{p}+\frac{k_{i}}{s}+k_{d} s
$$

where $\mathrm{k}_{\mathrm{p}}, \mathrm{k}_{\mathrm{i}}, \mathrm{k}_{\mathrm{d}}$ are the gains for the proportional, integral, and derivative terms, respectively.

The closed-loop system transfer function is

$$
\frac{x_{o}}{r}=\frac{G_{q}(s) G(s)}{1+P I D(s) G(s)}
$$

The key point is to choose suitable parameters of the 2-DOF PID controller. Firstly, mask the $\mathrm{G}_{\mathrm{q}}(\mathrm{s})$ block, the system becomes a 1-DOF PID control system. Determine the suitable parameters of the 1-DOF PID controller, $\mathrm{k}_{\mathrm{p}}, \mathrm{k}_{\mathrm{i}}$ and $\mathrm{k}_{\mathrm{d}}$, by using trial and error method. Then, add the $\mathrm{G}_{\mathrm{q}}(\mathrm{s})$ block in the system which is shown in Fig. 2. Set the $\mathrm{q}_{2}=0$ (The design is to be carried out with the feedforward parameter $\mathrm{q}_{2}=0$ ). For keeping the system`s tracking performance, set $\Delta \mathrm{x}_{\mathrm{o}} / \Delta \mathrm{r}=1$ at $\mathrm{s}=0$. Then following (17) obtains

$$
q_{1}=k_{i}
$$

So set the $\mathrm{q}_{1}$ by (18). Finally, increase and fine turn the $\mathrm{q}_{2}$ to get an appropriate speed of the response.

\section{SiMULATION AND EXPERIMENTAL RESULTS}

The magnetic levitation ball system is implemented both in simulation and the real experimental setup to further validate the effectiveness of the proposed control strategy. The 2-DOF PID controller Simulink model is shown in Fig. 3.

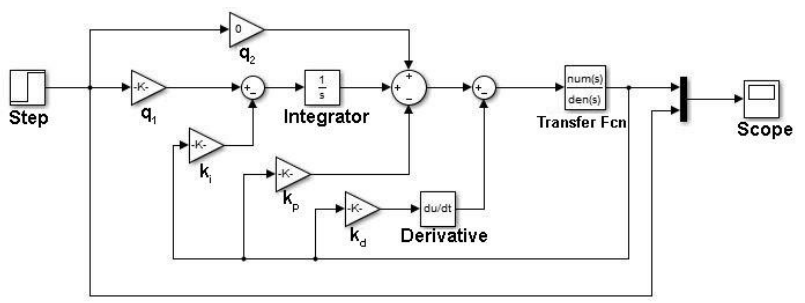

Fig. 3. The Simulink model of the 2-dof PID controller.

Firstly, Let $\mathrm{q} 2=0, \mathrm{q} 1=1$ to make the Simulink model of the 2-DOF PID controller become 1-DOF PID controller. Then the best parameters of the $\mathrm{kp}$, ki and $\mathrm{kd}$, via trial and error method, can be found. In this case, $\mathrm{kp}=10, \mathrm{ki}=4$ and $\mathrm{kd}=0.125$ are used. However from the Fig. 4, it can be seen that the step response generates a large overshoot at the beginning. It means as for a high nonlinear system, the normal 1-DOF PID controller cannot achieve a satisfactory result.

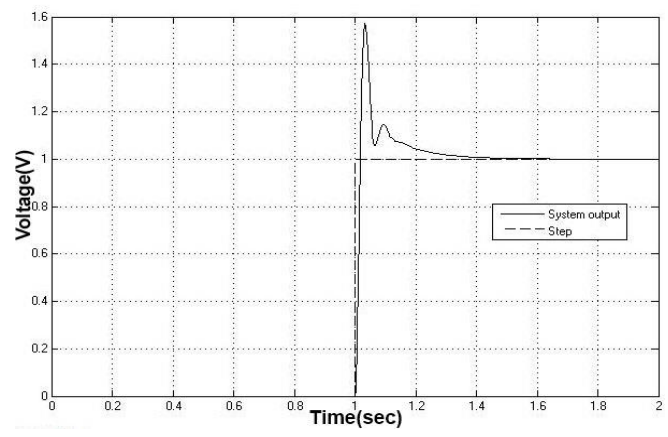

Fig. 4. Normal PID controller at step response.

Then, try to find the parameters for 2-DOF PID controller. By following (18), $\mathrm{q}_{1}=4$. Fig.5 shows that, the 2-DOF PID with $\mathrm{q}_{2}=0$ has minimal overshoot, however the rise time is the longest. 


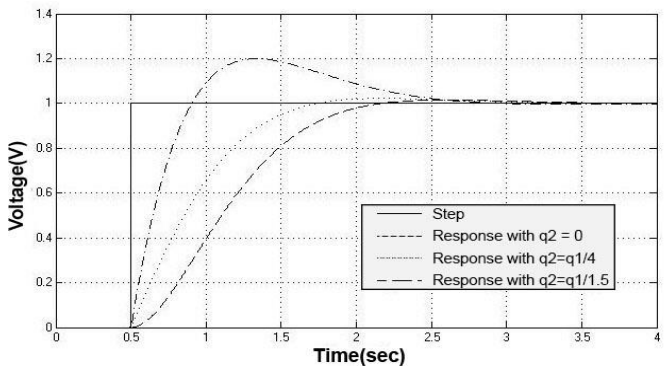

Fig. 5. 2-DOF PID controller at step response.

Then, It is verified through simulations that with an increase in $\mathrm{q}_{2}$, the speed of the response increases and with the choice of $\mathrm{q}_{2}=\mathrm{q}_{1} / 4$ it is found through simulations that the system's responses are quicker. And the rise time is much shorter, but the overshoot is almost same as the curve which is $\mathrm{q}_{2}=0$. Continue to increase the system responses by setting $\mathrm{q}_{2}=\mathrm{q}_{1} / 1.5$. The rise time of the curve is shorter than before, however the overshoot is too much lager.

Real experiment structure is shown in Fig. 6. The Hilink board [10] is used as the control board connecting the Matlab/Simulink control model and the real magnetic levitation ball system. This platform enables Matlab/Simulink simulate and control the system in real-time. Hilink Simulink Library provides the A/D and DA blocks.

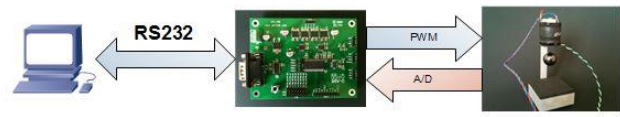

Fig. 6. The experiment platform.

Fig.7 shows the Simulink model with a 2-DOF PID controller for the real magnetic levitation ball system where the Hilink blocks $\mathrm{A} 0$ and $\mathrm{H} 0$ are used to represent the analog input and output respectively. There is a reference voltage input block which is used for deciding the position of the ball. A sinusoidal signal block is used for generating sinusoidal signal and adds this signal to reference voltage signal. By comparing the difference voltage between the reference voltage and the A0 block input voltage to get the input $r$ of the controller. Then the 2-DOF PID controller generates corresponding control voltage.

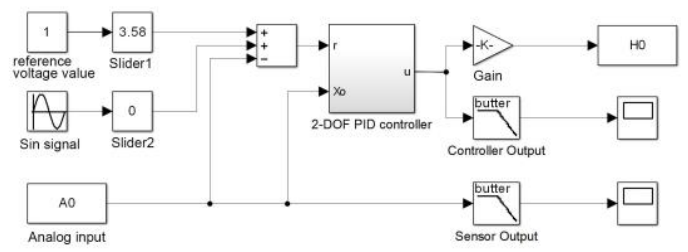

Fig. 7. Implementation of the 2-DOF PID controller.

When using one electromagnet which is connected with the output block $\mathrm{H} 0$ to attract the ball, the reference voltage value of the input is only from $3.5 \mathrm{~V}$ to $3.6 \mathrm{~V}$. And if it continues to increase the distance value (reference voltage value), the ball will fall off. In order to measure the movement range of the ball, when the ball suspending at the $3.58 \mathrm{~V}$, an extra sinusoidal signal, of which the amplitude is 1 , and the frequency is $1 \mathrm{~Hz}$, is added on the reference voltage signal. Then via the Slider Gain block to scale the sinusoidal signal to 0.1 times, the ball can move between around the $3.5 \mathrm{~V}$ and $3.6 \mathrm{~V}$. However if the sinusoidal signal is amplified more than 0.1 times, the ball drops off. Fig. 8 shows that the input voltage value of the A0 block is a sinusoidal curve signal. It means the ball is reciprocating, and the maximal movement range of the ball is from $3.50 \mathrm{~V}$ to $3.6 \mathrm{~V}$. At this time, the curve has a little distortion at the vertex. So the system is almost out of control.

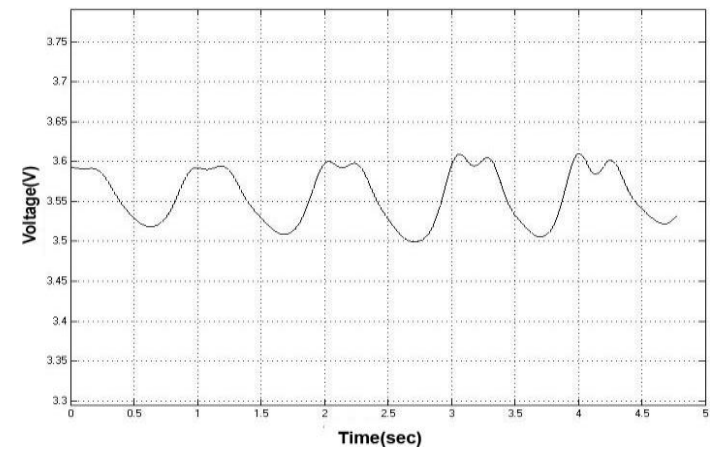

Fig. 8. Sensor output voltage with one electromagnet.

Then, another same standard electromagnet is set under the ball, and connected to the $\mathrm{H} 1$ pin of the Hilink board. Thus two electromagnets can cooperate to make the ball suspend, which is shown in Fig. 9. The H1 block and H0 block are all connected to the outputs of the 2-DOF PID controller, and another Slide Gain block is added on the H1 output signal to regulate the output voltage of the $\mathrm{H} 1$. The initial value of this Slide Gain block is 0.48 times, if this value is less than 0.48 , the output voltage of $\mathrm{H} 1$ is not big enough to affect the system .

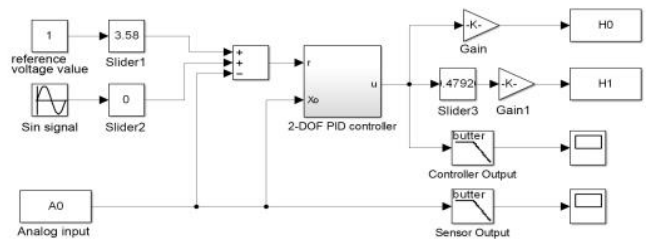

Fig. 9. The 2-DOF PID controller with two outputs.

In order to test the range of the movement, change the reference voltage value to make the ball suspend at the $3.58 \mathrm{~V}$ of the sensor output. Then move the electromagnet $\mathrm{L}_{2}$ to make $\mathrm{x}_{1}=\mathrm{x}_{2}$, the ball levitates at the centre of the two electromagnets. The controller used is shown in Fig. 9, and all other parameters are same as the last experiment.

Fig. 10 and Fig.11 show that, with the same parameters, the new structure of the system is more stable. The sensor output voltage curve is not distorted. It means the new structure is more stable than one-electromagnet structure. 


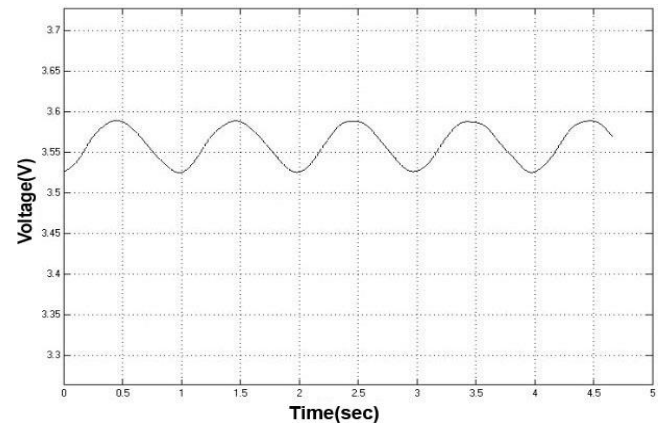

Fig. 10. Sensor output voltage using two electromagnets.

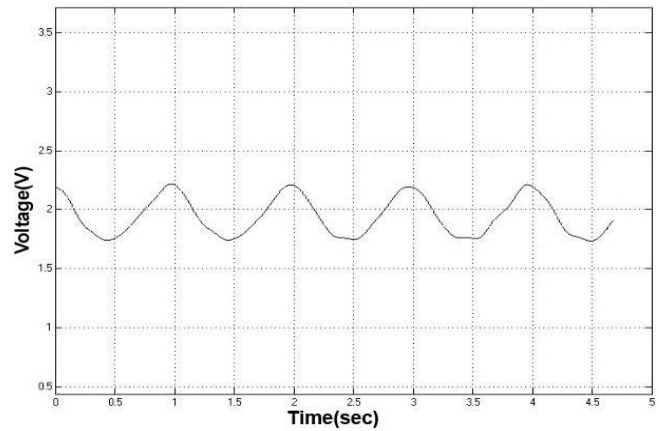

Fig. 11. Controller output voltage using two electromagnets.

Then continue to amplify the sinusoidal signal via increasing the value of the Slide Gain block, the range of the ball movement can still increase.

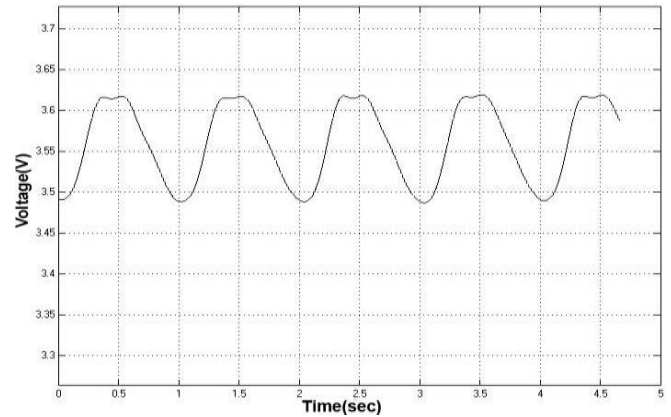

Fig. 12. Sensor output voltage using two electromagnets with increased gain of sinusoidal signal.

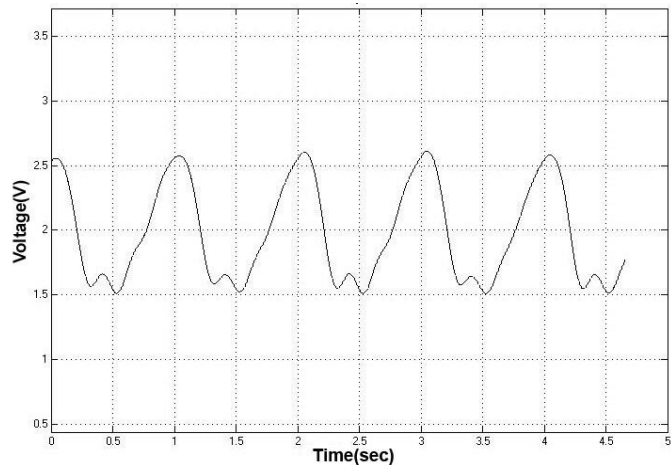

Fig. 13. Controller output voltage using two electromagnets with increased gain of sinusoidal signal.
Fig. 12 shows the output of the A0 block when the input of the sinusoidal signal is amplified to 0.2 times. And the ball is moving between $3.48 \mathrm{v}$ and $3.62 \mathrm{v}$. At this time, the curve of the sensor output voltage is distorted.

Fig. 13 shows the controller output voltage, It is also a sinusoidal curve signal however it is shown that the system is almost out of control.

Then, increase the value of the Slide Gain block, which is connected to the $\mathrm{H} 1$ block, to 0.57 times, so that the voltage of the $\mathrm{H} 1$ output is increased. The distortion of the sensor output voltage curve disappears, which is shown in Fig. 14. And Fig. 15 shows that the controller works better than before.

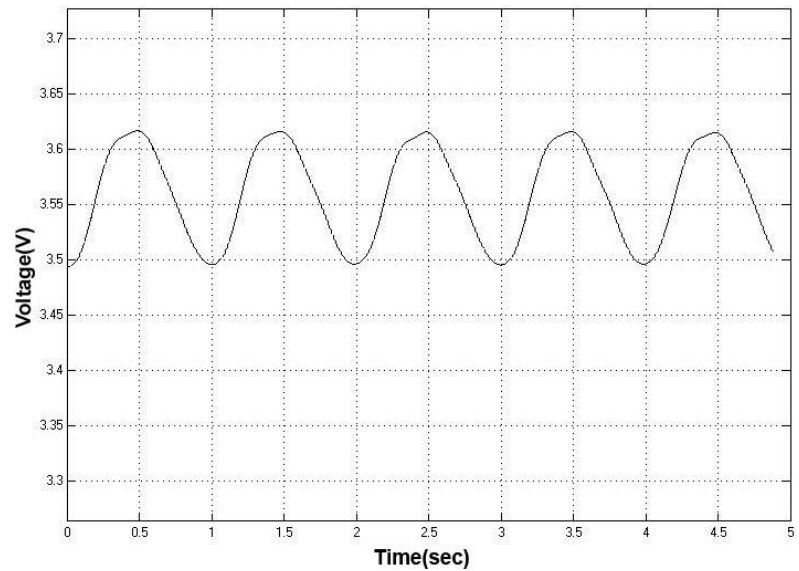

Fig. 14. Sensor output voltage using two electromagnets with increased H1 output.

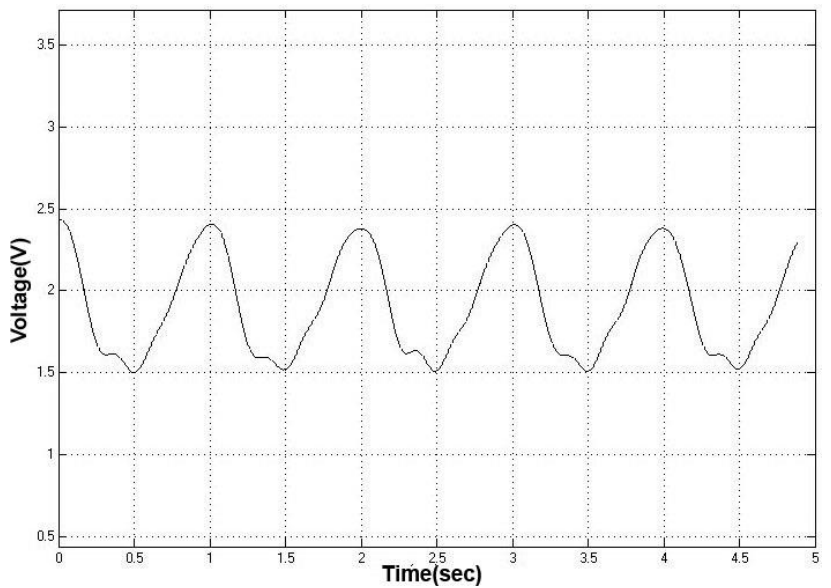

Fig. 15. Controller output voltage using two electromagnets with increased H1 output.

Repeat the above steps, when the sinusoidal signal is amplified to 0.33 times, and $\mathrm{H} 1$ is amplified to 0.76 times, Fig. 16 and Fig. 17 show the sensor output voltage curve and controller output voltage curve, which mean the movement range of the ball is from $3.46 \mathrm{~V}$ to $3.64 \mathrm{~V}$, and the system is still controlled well. So using new structure can effectively increase the range of the ball's movement and the system is more stable. 


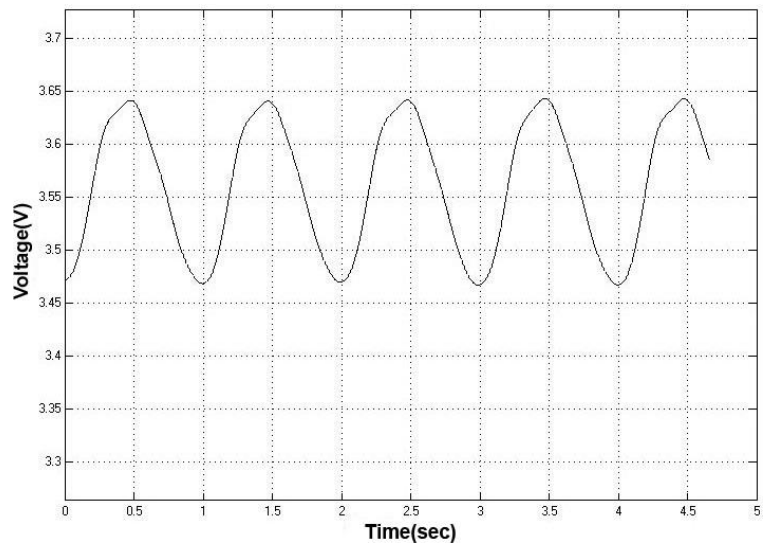

Fig. 16. Sensor output voltage using two electromagnets with increased movement range.

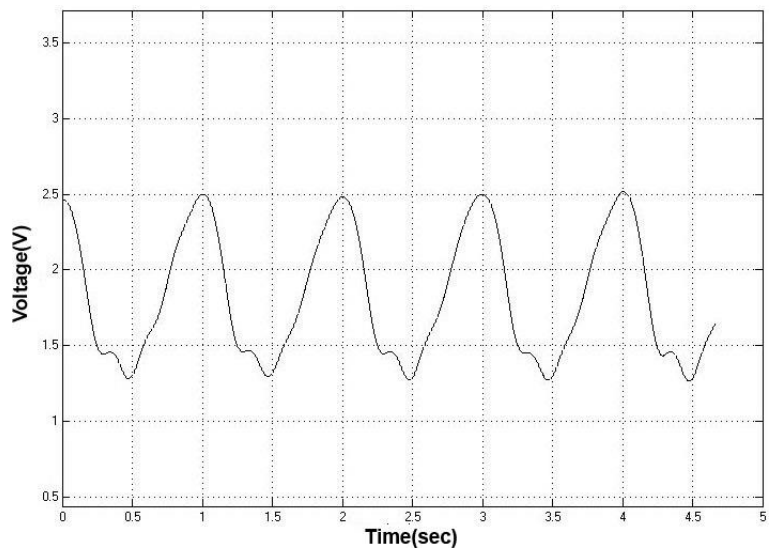

Fig. 17. Controller output voltage using two electromagnets with increased movement range.

\section{CONCLUSION}

A two-electromagnet magnetic levitation ball system is proposed in this paper and a 2-DOF PID controller is applied. This new structure of the magnetic levitation ball system shows better performance than previous magnetic levitation ball systems. From the simulation and real experiment results it can be seen that using two electromagnets can make a ball levitate and can not only increase the balance range of the ball, but also make the system more stable.

A 2-DOF PID controller has also been proposed in this paper for controlling the real magnetic levitation ball system. Although it has been noted that the conventional PID controller depends on the system parameters, the superior robustness of the 2-DOF configuration can be achieved in real-time by properly setting the feed-forward term.

Increasing the balance range of the ball means the magnetic levitation ball system can apply in more fields. At the same time, a simple and effective controller, like 2-DOF PID controller, is more economical and occupies less computational resources, so there are more advantages in practical application than other controllers.

\section{REFERENCES}

[1] Wong, T. H. "Design of a magnetic levitation control system-an undergraduate project." IEEE Transactions on Education 29.4 (1986): 196-200.

[2] El Hajjaji, Ahmed, and M. Ouladsine. "Modeling and nonlinear control of magnetic levitation systems." IEEE Transactions on Industrial Electronics 48.4 (2001): 831-838.

[3] 松村文夫, and 山田外史. "A Control Method of Suspension Control System by Magnetic Attractive Force." 電気学会論文誌. B 94.11 (1974): 567-574.

[4] Jayawant, B. V., and D. P. Rea. "New electromagnetic suspension and its stabilisation." Electrical Engineers, Proceedings of the Institution of 115.4 (1968): 549-554.

[5] Laithwaite, E. R. "Electromagnetic levitation." Electrical Engineers, Proceedings of the Institution of 112.12 (1965): 2361-2375.

[6] Al-Muthairi, N. F., and M. Zribi. "Sliding mode control of a magnetic levitation system." Mathematical Problems in Engineering 2004.2 (2004): 93-107.

[7] Lee, Hyung-Woo, Ki-Chan Kim, and Ju Lee. "Review of maglev train technologies." Magnetics, IEEE Transactions on 42.7 (2006): 1917 1925.

[8] Hoshi, Hideo, Tadahiko Shinshi, and Setsuo Takatani. "Third generation Blood Pumps With Mechanical Noncontact Magnetic Bearings." Artificial organs 30.5 (2006): 324-338.

[9] Song, Chun-sheng, et al. "Dynamic modeling of magnetic suspension isolator using artificial neural network: a modified genetic approach." Journal of Vibration and Control 19.6 (2013): 847-856.

[10] Lee, Hyung-Woo, Ki-Chan Kim, and Ju Lee. "Review of maglev train technologies." Magnetics, IEEE Transactions on 42.7 (2006): 19171925 .

[11] Joo, SungJun, and Jin H. Seo. "Design and analysis of the nonlinear feedback linearizing control for an electromagnetic suspension system." Control Systems Technology, IEEE Transactions on 5.1 (1996): 135144.

[12] Zhao, Feng, Shiou C. Loh, and Jeff A. May. "Phase-space nonlinear control toolbox: The maglev experience." Hybrid Systems V. Springer Berlin Heidelberg, 1999. 429-444.

[13] Mohamed, A. M., et al. "Q-parametrization//spl mu/-control of an electromagnetic suspension system." Control Applications, 1997., Proceedings of the 1997 IEEE International Conference on. IEEE, 1997.

[14] Fujita, M. A. S. A. Y. U. K. I., Fumio Matsumura, and Kenko Uchida "Experiments on the $\mathrm{H} \infty$ disturbance attenuation control of a magnetic suspension system." Decision and Control, 1990., Proceedings of the 29th IEEE Conference on. IEEE, 1990.

[15] Salim, Tania Tariq, and Vedat Mehmet Karsli. "Control of Single Axis Magnetic Levitation System Using Fuzzy Logic Control." International Journal of Advanced Computer Science and Applications 4.11 (2013).

[16] Lairi, Mostafa, and Gerard Bloch. "A neural network with minimal structure for maglev system modeling and control." Intelligent Control/Intelligent Systems and Semiotics, 1999. Proceedings of the 1999 IEEE International Symposium on. IEEE, 1999.

[17] Ghosh, Arun, et al. "Design and implementation of a 2-DOF PID compensation for magnetic levitation systems." ISA transactions 53.4 (2014): 1216-1222.

[18] Lin, Chih-Min, Chih-Min Lin, and Chun-Wen Chen. "SoPC-based adaptive PID control system design for magnetic levitation system." Systems Journal, IEEE 5.2 (2011): 278-287.

[19] Gibbs, W. W. "Antigravity glitter build a levitating disco-ball nightlight; bell-bottom pants optional." Spectrum, IEEE 51.8 (2014): 23-25.

[20] WANG, Weijie, et al. "Two-degrees-of-freedom PID controller tuning method [J]." Journal of Tsinghua University (Science and Technology) 11 (2008): 020.

[21] Gorez, Raymond. "New design relations for 2-DOF PID-like control systems." $\quad$ Automatica $\quad 39.5 \quad$ (2003): 901-908 